\title{
RECONOCIMIENTO DE ALIMENTOS VEGETALES: CARACTERIZACIÓN MICRO-GRÁFICA DEL GRANO DE AVENA ${ }^{1}$
}

\author{
N.O. CRIVARO ${ }^{2}$, N.M. APÓSTOLO ${ }^{3}$, S. CALLONI ${ }^{2}$, E. LORENZO $^{3}$, L. VIÑALES ${ }^{2}$, P. GUALDIERI ${ }^{2}$
}

\section{RESUMEN}

Se estudia la estructura micrográfica del grano de seis variedades de avena con la finalidad de su caracterización, para desarrollar parámetros de identificación en alimentos elaborados con la misma y, consecuentemente, determinar su autenticidad, contribuyendo a optimizar la producción, la comercialización y el consumo del cereal y sus derivados. El diseño experimental consistió en el estudio micrográfico de los granos vestidos y desnudos efectuando un análisis morfológico mediante observación con lupa binocular y fotografía, ultraestructural utilizando microscopio electrónico de barrido, micrográfico y micrométrico, empleando el sistema de video microscopia digitalizado y software adecuado. Dada su variabilidad natural, los estudios se efectuaron durante tres temporadas consecutivas sobre muestras cosechadas de variedades procedentes de cultivos de semillas certificadas, y sobre alimentos procesados (avena arrollada y salvado de avena comerciales). Los resultados consistieron en diseños micrográficos, y en valores micrométricos de gránulos de almidón relacionados, además, en modelos matemáticos. En todos los casos se validó estadísticamente. Como parámetros micrográficos de caracterización se seleccionaron las estructuras diferenciales, que revelaron una presencia constante en el vegetal y resistieron los tratamientos tecnológicos, y las características y dimensiones del almidón.
\end{abstract}

Palabras llave: avena, cereales, micrografía.

\section{RESUMO}

RECONHECIMENTO DE ALIMENTOS VEGETAIS: CARACTERIZAÇÃO MICROGRÁFICA DO GRÃO DE AVEIA. Este trabalho estuda a estrutura micrográfica do grão de seis variedades de aveia com a finalidade de sua caracterização para desenvolver parâmetros de identificação em alimentos elaborados com a mesma e, conseqüentemente, determinar sua genuinidade, contribuindo para otimizar a produção, a comercialização e o consumo do cereal e seus derivados. O experimento consistiu no estudo micrográfico dos grãos com casca e sem casca, efetuando análise morfológica mediante observação com lupa binocular e fotografia ultra-estrutural utilizando microscópio eletrônico de varredura micrográfico e micrométrico, empregando sistema de vídeo-microscopia digitalizada e software adequado. Dada sua variabilidade natural, os estudos se efetuaram durante três temporadas consecutivas sobre as amostras recolhidas de variedades procedentes de cultivos de sementes certificadas e sobre alimentos processados (aveia enrolada e salvado de aveia comerciais). Os resultados consistiram em projetos micrográficos e em valores micrométricos de grânulos de amido, relacionados, além disso, em modelos matemáticos. Em todos os casos se convalidou estatisticamente. Como parâmetros micrográficos de caracterização, se selecionaram as estruturas diferenciais que revelaram uma presença constante no vegetal e resistiram aos tratamentos tecnológicos e as características e dimensões do amido.

Palavras chave: aveia, cereais, micrografia.

\section{INTRODUCCIÓN}

El 80\% de la población mundial utiliza productos naturales de origen vegetal en la preservación y cuidado de su salud. La caracterización de la autenticidad de materias primas vegetales y de alimentos derivados constituye un problema de difícil resolución analítica. Esto se traslada no sólo a los organismos oficiales sino también a productores, industriales y a los consumidores, que deben conformarse con lo que se les ofrece, pues la legislación que podría servir como referente sobre calidad, autenticidad y seguridad de lo que consumen, muchas veces no les resulta conocida o está desactualizada.

Por otra parte, se encuentra en vigencia el Acuerdo de Marrakech ${ }^{[1]}$, aplicable en países en vías de desarrollo desde enero de 2000, que entre sus obligaciones se contemplan normas de propiedad intelectual como, por ejemplo, la protección de las Indicaciones Geográficas y de las Deno-

\footnotetext{
${ }^{1}$ Recebido para publicação em 8/12/2004. Aceito para publicação em 28/04/2006 (001452)

${ }^{2}$ Departamento de Tecnología y

${ }^{3}$ Departamento de Ciencias Básicas. Universidad Nacional de Luján. Cruce Rutas 5 y 7 - (6700) Luján. Provincia de Buenos Aires. Argentina. E-mail:apostolo@mail.unlu.edu.ar
}

minaciones de Origen. Representaría un beneficio para los productores y para los países en general estar preparados para poder establecer y fiscalizar la procedencia de los alimentos y/o sus materias primas.

Por eso hoy en día se plantea la necesidad de disponer de herramientas para una caracterización segura del origen de esos productos, ya que los datos y antecedentes obtenidos de la misma garantizarán la credibilidad del alimento a través de la autorización otorgada por los organismos oficiales.

La avena es un cereal que además de emplearse como forraje y en la industria cosmética [2, 5], presenta un consumo en franca expansión como alimento, ya sea a través del uso tradicional (cereales para desayuno, derivados farináceos) o de su inclusión en productos a los que confiere el carácter de "funcionales", bajo formas que lindan con la dietoterapia. En este sentido, se la incorpora en diversos preparados comerciales que han ganado el mercado a nivel mundial, no sólo como fitonutriente, sino por la adjudicación de algunas propiedades tales como hipocolesterolemizante, preventiva de cardiopatías, normalizadora de estados fisiológicos alterados (estrés, disminución de la libido, disminución 
de la potencia sexual masculina, ansiedad, etc.). La actividad en la prevención de cardiopatías es reconocida por la FDA que, en 1997, autorizó a los productores de alimentos a consignar en los rótulos de los mismos que "las dietas con alto contenido de fibra soluble procedente del grano entero de avena puede reducir el riesgo de las enfermedades cardíacas" [6], aunque los países de la Unión Europea aún no se han puesto de acuerdo con respecto al tema.

Por otra parte, tal como ocurre con otros alimentos, existe profusa información sobre su composición química y características fisicoquímicas, producción, cultivo, rendimiento y otros aspectos agronómicos, pero presentada de manera muchas veces incompleta o como colaboración aislada [7, 21]. Esto no resulta adecuado para la caracterización de la especie en productos derivados, ya que los valores de los parámetros desarrollados pueden invalidarse fácilmente por el empleo de adulterantes baratos de fácil acceso comercial.

Por lo expresado hemos desarrollado este estudio con el fin de ampliar el conocimiento sobre composición y características morfoanatómicas de seis variedades de avena, para contribuir a su reconocimiento y desarrollar un sistema más seguro y accesible de determinación de genuinidad de alimentos derivados.

\section{EXPERIMENTAL}

La metodología utilizada tiene antecedentes en desarrollos previos de sistemas de caracterización que hemos realizado para otros alimentos vegetales [22, 24].

Para este estudio se analizaron cuatro variedades argentinas originadas en programas de mejoramiento del sur de la provincia de Buenos Aires (INTA Bordenave y Chacra Barrow, y Facultad de Agronomía de la Universidad Nacional de la Plata): Millauquen INTA, Bonaerense INTA Maja, Bonaerense INTA Calén y Máxima INTA; y: Suregrain (variedad de origen estadounidense) y Polaris INTA (introducida de Uruguay). El cultivo de las diferentes variedades se realizó en parcelas experimentales del campo de la Universidad Nacional de Luján (Provincia de Buenos Aires), a partir de semillas certificadas.

El material vegetal tipo consistió en muestras de granos maduros colectados durante tres cosechas consecutivas.

Como material comparativo se utilizó avena arrollada y salvado de avena comerciales, con la finalidad de analizar la influencia de los procesos tecnológicos sobre las variables estudiadas.

El estudio morfológico consistió en la observación y fotografía de los granos vestidos y desnudos de cada variedad y de las muestras comerciales, utilizando lupa binocular Zeiss.

El estudio microscópico y micrométrico se realizó empleando sistema de videomicroscopia digitalizado y software micrométrico OPTIMAS 6.2 en microscopio óptico UNICO (MO). Para la preparación de las muestras se siguió la metodología tradicional $[25,26]$. Se realizó la prueba microquímica para almidón con reactivo de Lugol. Los datos (largo y ancho de amiloplastos compuestos y subunidades) fueron obtenidos por lectura de campos al azar en tres preparados por muestra $(n=30)$.

El estudio ultraestructural se basó en la observación y fotografía de la superficie y cortes transversales de las diferentes partes del grano vestido y desnudo de cada variedad, y de la superficie de las muestras comerciales, utilizando microscopio electrónico de barrido (MEB) Jeol JEOR T100 (Servicio de Microscopia Electrónica, Museo de Ciencias Naturales, La Plata, Argentina).

Los resultados micrométricos se analizaron por ANOVA de un solo factor y comparación múltiple de medias (test Duncan). Además se determinaron desvío standard, coeficiente de variación porcentual e intervalos de confianza para el $95.5 \%(z=2)$. Los datos fueron sometidos a análisis regresivo.

\section{RESULTADOS Y DISCUSIÓN}

El grano de avena vestido está cubierto por un par de glumelas (lenma o externa y pálea o interna). La lenma es aristada, pajiza, envuelve la pálea. La pálea es delgada, pajiza y cubre el grano (Figura 1 A). Todas las variedades estudiadas muestran granos vestidos con características y color similar (ocre) aunque Millauquen INTA es más oscura.

AL MEB se observa que ambas superficies de las glumelas son glabras y tienen células alargadas. La epidermis abaxial (cara inferior) de la lenma tiene papilas y aguijones, y epidermis adaxial (cara superior) es lisa con estomas (Figuras $1 \mathrm{~B}, \mathrm{C}$ ). La superficie de la palea es lisa (Figura $1 \mathrm{D}$ ).

$\mathrm{El}$ grano desnudo, es decir, libre de glumelas, muestra en todas las variedades superficie muy pilosa, siendo Millauquen INTA la de mayor densidad de pelos, principalmente en la parte posterior del grano (Figuras $1 \mathrm{E}, \mathrm{F}$ ). También el germen está totalmente cubierto con estos pelos unicelulares, de pared lisa y terminación aguda. (Figura 1 E).

La observación de los cortes transversales del grano con MEB evidencia, debajo de la cubierta seminal, una capa externa de células con gránulos de aleurona. El endosperma del grano, observado al MO y MEB, muestra células ricas en amiloplastos compuestos formados por numerosas subunidades poligonales (gránulos de almidón). (Figuras $1 \mathrm{G}-J$ ).

Mediante el ANOVA y test de Duncan se determinaron diferencias significativas $(\mathrm{P}=0.05)$ entre variedades en las dimensiones de los amiloplastos completos y de las subunidades (Tabla 1).

Se observa que las variedades Bonaerense INTA Calen, Bonaerense INTA Maja, Máxima INTA y Suregrain presentan los amiloplastos completos de menores dimensiones, mientras que Millauquen INTA muestra los de mayor largo y ancho. Por otra parte, las mayores dimensiones en las subunidades del amiloplasto se encontraron en Máxima INTA (Tabla 1). 

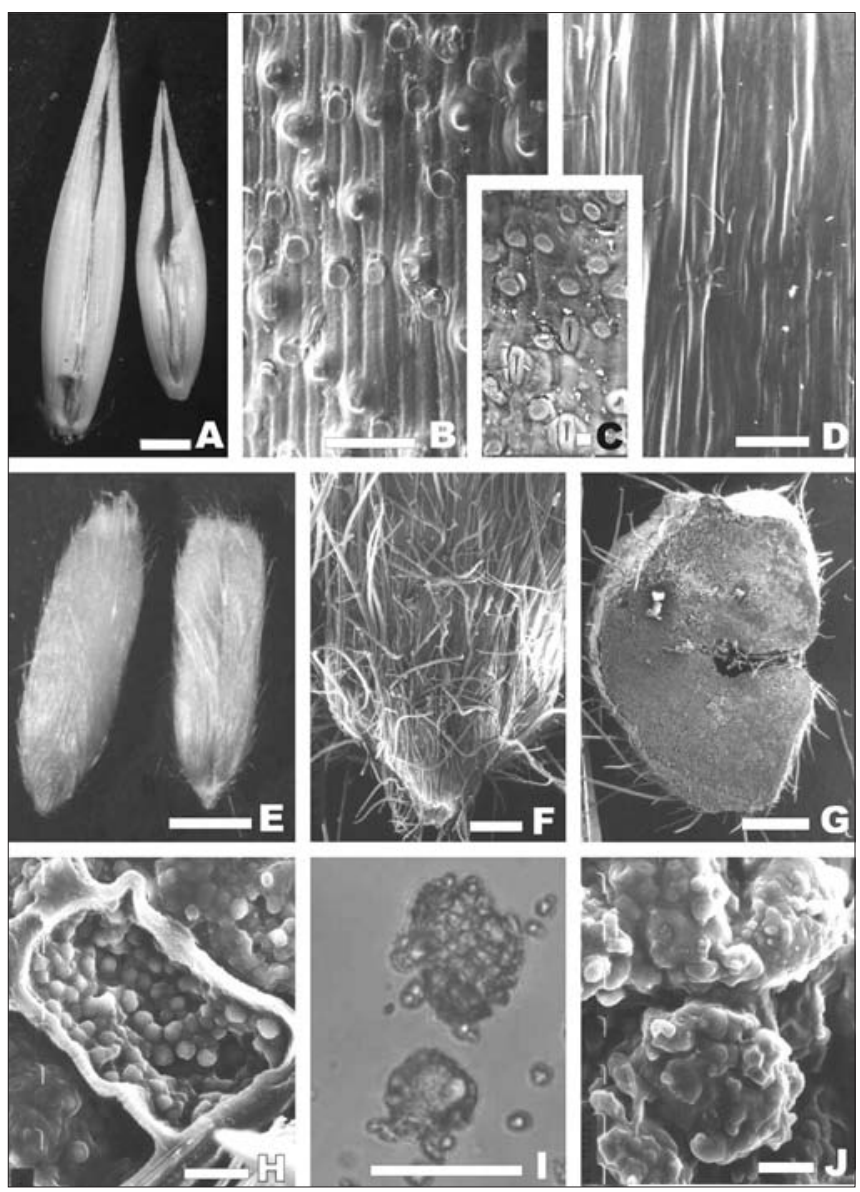

FIGURA 1 - Granos de avena: A, grano vestido de Bonaerense INTA Maja; B, epidermis abaxial de la lenma de Bonaerense INTA Calen (MEB); C, epidermis adaxial de la lenma de INTA Polaris (MEB); D, epidermis de la palea de INTA Polaris; E, grano desnudo de Maxima INTA; F, vista superficial del grano desnudo de Millauquen INTA, detalle del germen (MEB); G, corte transversal del grano desnudo de Bonaerense INTA Calen (MEB); H, célula de la capa de aleurona del grano de Bonaerense INTA Calen (MEB); I, amiloplastos compuestos del endosperma de Bonaerense INTA Calen (MO); J, amiloplastos compuestos de INTA Polaris.

Barras: A, E: $2 \mathrm{~mm}$; F, G: $500 \mu \mathrm{m}$; B, D, I: $50 \mu \mathrm{m}$; H, J, C: $10 \mu \mathrm{m}$

TABLA 1 - Dimensiones de los amiloplastos de granos de avena (ANOVA y test de Duncan).

\begin{tabular}{|c|c|c|c|c|}
\hline \multirow[b]{2}{*}{ Variedad } & \multicolumn{2}{|c|}{ Amiloplasto completo } & \multicolumn{2}{|c|}{ Subunidades } \\
\hline & Largo $(\mu)$ & Ancho $(\mu)$ & Largo $(\mu)$ & Ancho $(\mu)$ \\
\hline $\begin{array}{l}\text { Millauquén } \\
\text { INTA }\end{array}$ & $41.27 \mathrm{a}^{(1)}$ & $33.09 \mathrm{a}$ & $8.44 a b$ & $7.07 \mathrm{a}$ \\
\hline Polaris INTA & $36.89 \mathrm{ab}$ & $31.07 \mathrm{a}$ & $8.50 a b$ & $6.72 \mathrm{a}$ \\
\hline $\begin{array}{l}\text { Bonaerense } \\
\text { INTA Calén }\end{array}$ & $30.98 \mathrm{~b}$ & $24.21 \mathrm{~b}$ & $7.22 \mathrm{c}$ & $6.10 a b$ \\
\hline $\begin{array}{l}\text { Bonaerense } \\
\text { INTA Maja }\end{array}$ & $31.05 \mathrm{~b}$ & $23.55 \mathrm{~b}$ & $7.45 \mathrm{bc}$ & $6.19 a b$ \\
\hline Máxima INTA & $32.63 \mathrm{~b}$ & $26.76 \mathrm{~b}$ & $8.83 \mathrm{a}$ & $6.44 \mathrm{a}$ \\
\hline Suregrain & $32.87 \mathrm{~b}$ & $24.03 \mathrm{~b}$ & $7.24 \mathrm{c}$ & $5.46 \mathrm{~b}$ \\
\hline
\end{tabular}

En el análisis estadístico elemental (Tabla 2) la tendencia central en la distribución se ubicó por la media, la dispersión en valores absolutos por la desviación standard y en valores relativos por el coeficiente de variación porcentual. Para todos los casos se determinó el intervalo de confianza para $z=2$. Puede observarse, tanto para amiloplasto completo como para subunidades, zonas de valores que son comunes para diferentes variedades, aunque el ANOVA y el test de Duncan revelan diferencias significativas en ambos casos.

El análisis regresivo consistió en la correlación y la regresión lineal entre las dimensiones obtenidas por la micrometría para los amiloplastos de cada variedad, lo que permitió formular modelos matemáticos que las relacionan entre sí y que deberán cumplirse, con las adecuaciones que resulten necesarias en el caso de que sufran alteraciones por la tecnología empleada, en los alimentos derivados que sean genuinos (Tabla 3).

Los valores de los coeficientes de correlación $r$ y de regresión $\mathrm{r}^{2}$ evidencian que, para el amiloplasto completo, existe considerable constancia en la relación entre las dimensiones largo y ancho, es decir, homogeneidad en su forma. Para las subunidades, sólo la variedad Bonaerense INTA Calén presenta esta característica. La hipótesis nula fue que no existía correlación real entre las variables, por lo que para $F$ se consideró $\mathrm{H}_{0}: \beta=0 \mathrm{y} \mathrm{H}_{1}: \beta \neq 0$. Los valores $\mathrm{F}$ críticos se obtuvieron de tablas para prueba bilateral cuando $\alpha=0.05$. Los resultados de este análisis confieren validez a los modelos matemáticos desarrollados.

Las muestras de avena arrollada y salvado de avena comerciales se encuentran contempladas en los artículos 655 y 655 bis, respectivamente, del Código Alimentario Argentino [27] . En ellos se establece que los granos deben estar "libres de tegumento", es decir, que se deben extraer las glumelas y pelar la superficie de la cubierta seminal quedando el grano desnudo (Figuras $2 \mathrm{~A}, \mathrm{~B}$ ), y someterse a tratamientos térmicos que aseguren la inactivación de las enzimas.

Se analizaron muestras de dos marcas comerciales diferentes ("X" e "Y") de cada uno de los productos. Los resultados obtenidos demostraron que:

a. a pesar del procesamiento tecnológico sufrido, se conservan las características morfoanatómicas, ultraestructurales y micrométricas originales de los amiloplastos, glumelas y gránulos de aleurona (Figuras. 2 C-H; 3 A-D);

b. las muestras de avena arrollada de la marca "X" revelaron la presencia de elementos histológicos característicos de los constituyentes citados (Figuras. $2 \mathrm{C}-\mathrm{H}$ ) y

c. en las muestras restantes (avena arrollada de la marca "Y", y salvado de avena de las marcas "X" e "Y") no se observaron elementos de glumelas (Figuras. 2 C-E, G-H; 3 A-D). 
TABLA 2 - Estadísticos elementales de las dimensiones de los amiloplastos de semillas de avena.

\begin{tabular}{|c|c|c|c|c|c|c|}
\hline \multicolumn{7}{|c|}{ AMILOPLASTO COMPLETO } \\
\hline \multirow{2}{*}{ Variedad } & \multicolumn{3}{|c|}{ Largo } & \multicolumn{3}{|c|}{ Ancho } \\
\hline & $\mathbf{S}^{(1)}$ & $\mathrm{CV}^{(2)}$ & $\mathrm{IC}^{(3)}(\mu)$ & s & CV\% & IC $(\mu)$ \\
\hline Millauquen INTA & 11,04 & 26,75 & 19,19 a 63,35 & 8,24 & 24,96 & 16,54 a 49,52 \\
\hline Polaris INTA & 8,38 & 22,72 & 20,13 a 53,65 & 7,98 & 25,68 & 15,11 a 47,04 \\
\hline Bonaer. INTA Calén & 7,46 & 24.08 & 16,06 a 45,90 & 4,31 & 17,81 & 15,58 a 32,83 \\
\hline Bonaer. INTA Maja & 7,76 & 24,99 & 15,53 a 46,57 & 4,70 & 15,14 & 21,65 a 40,45 \\
\hline Suregrain & 10,78 & 32,80 & 11,31 a 54,43 & 6,99 & 21,26 & 21,26 a 46,85 \\
\hline \multicolumn{7}{|c|}{ SUBUNIDADES } \\
\hline & \multicolumn{3}{|c|}{ Largo } & \multicolumn{3}{|c|}{ Ancho } \\
\hline & $\mathbf{s}$ & $\mathrm{CV} \%$ & IC $(\mu)$ & $\mathbf{s}$ & CV\% & IC $(\mu)$ \\
\hline Bonaer. INTA Maja & 1,51 & 20,28 & 4,43 a 10,48 & 1,16 & 18,34 & 3,92 a 8,47 \\
\hline Máxima INTA & 1,55 & 17,51 & 5,74 a 11,92 & 1,18 & 18,23 & 4,10 a 8,80 \\
\hline Suregrain & 1,57 & 21,63 & 4,11 a 10,38 & 1,25 & 22,84 & 2,97 a 7,96 \\
\hline
\end{tabular}

$\mathrm{n}=30$ por variedad

${ }^{(1)}$ desvío standard; ${ }^{(2)}$ coeficiente de variación porcentual; ${ }^{(3)}$ intervalo de confianza $(\mathrm{z}=2)$

TABLA 3 - Análisis regresivo de las dimensiones de los amiloplastos de semillas de avena.

\begin{tabular}{|c|c|c|c|c|c|c|c|}
\hline \multicolumn{8}{|c|}{ AMILOPLASTO COMPLETO } \\
\hline Variedad & $r^{(1)}$ & $r^{2(2)}$ & $\mathbf{S}^{(3)}$ & $a^{(4)}$ & $\mathbf{b}^{(5)}$ & $F^{(6)}$ & $\mathrm{Fc}^{(7)}$ \\
\hline Millauquen INTA & 0,85 & 0,72 & 6,01 & 3,79 & 1,13 & 45,974862 & 0,000002 \\
\hline Polaris INTA & 0,69 & 0,48 & 6,22 & 14,34 & 0,73 & 16,467329 & 0,000738 \\
\hline Bonaer.INTA Calén & 0,73 & 0,53 & 5,25 & 0,45 & 1,26 & 20,393668 & 0,000267 \\
\hline Bonaer.INTA Maja & 0,75 & 0,56 & 5,21 & 2,33 & 1,22 & 22,970687 & 0,000146 \\
\hline Máxima INTA & 0,82 & 0,68 & 4,53 & $-3,18$ & 1,34 & 38,206958 & 0,000008 \\
\hline Suregrain & 0,76 & 0,58 & 7,15 & 4,55 & 1,18 & 25,225938 & 0,000088 \\
\hline
\end{tabular}

\begin{tabular}{|c|c|c|c|c|c|c|c|}
\hline \multicolumn{8}{|c|}{ SUBUNIDADES } \\
\hline & $r$ & $r^{2}$ & $\mathrm{~S}$ & a & b & $F$ & Fc \\
\hline Millauquen INTA & 0,45 & 0,21 & 1,17 & 5,98 & 0,35 & 4,953497 & 0,038342 \\
\hline Polaris INTA & 0,44 & 0,20 & 1,37 & 5,97 & 0,38 & 4,650506 & 0,044068 \\
\hline Bonaer.INTA Calén & 0,76 & 0,58 & 0,96 & 2,40 & 0,79 & 26,253956 & 0,000060 \\
\hline Bonaer.INTA Maja & 0,60 & 0,37 & 1,24 & 2,47 & 0,81 & 10,963393 & 0,003672 \\
\hline Máxima INTA & 0,58 & 0,34 & 1,29 & 3,91 & 0,76 & 9,636900 & 0,005838 \\
\hline Suregrain & 0,15 & 0,02 & 1,59 & 6,23 & 0,18 & 9,420124 & 0,524632 \\
\hline
\end{tabular}

$\mathrm{Y}=\mathrm{f}(\mathrm{X}) ; \mathrm{Y}=$ largo, $\mathrm{X}=$ ancho; $\mathrm{n}=30$ por variedad

(1) coeficiente de correlación; ${ }^{(2)}$ coeficiente de regresión; ${ }^{(3)}$ error tipico; ${ }^{(4)}$ constante; ${ }^{(5)}$ coeficiente de X; ${ }^{(6)}$ razón de varianzas, valor experimental; ${ }^{(7)}$ razón de varianzas, valor crítico

\section{CONCLUSIONES}

Los resultados obtenidos permitieron determinar que:

a. los elementos histológicos típicos de las variedades consideradas que persisten prácticamente inalterados después de los tratamientos tecnológicos habituales para avena arrollada y salvado de avena, en las condiciones de este estudio, son (Figuras $2 \mathrm{C}-\mathrm{H}$; 3 A-D):

(1) lenmas glabras, con células alargadas, cara inferior con papilas y aguijones y cara superior lisa con estomas;

(2) palea delgada con ambas superficies glabras, con células alargadas;
(3) cubierta del grano desnudo con superficie muy pilosa, con pelos unicelulares, de pared lisa y terminación aguda;

(4) germen totalmente cubierto con esos pelos;

(5) capa de aleurona;

(6) amiloplastos compuestos y subunidades libres, positivos a la reacción de Lugol, con formas y dimensiones características;

b. en los alimentos genuinos no deben observarse restos de palea o lenma ni pelos en cubierta seminal y germen, pues indicarían menor calidad de los mismos por no haber sufrido la extracción de glumelas y/o el pelado 

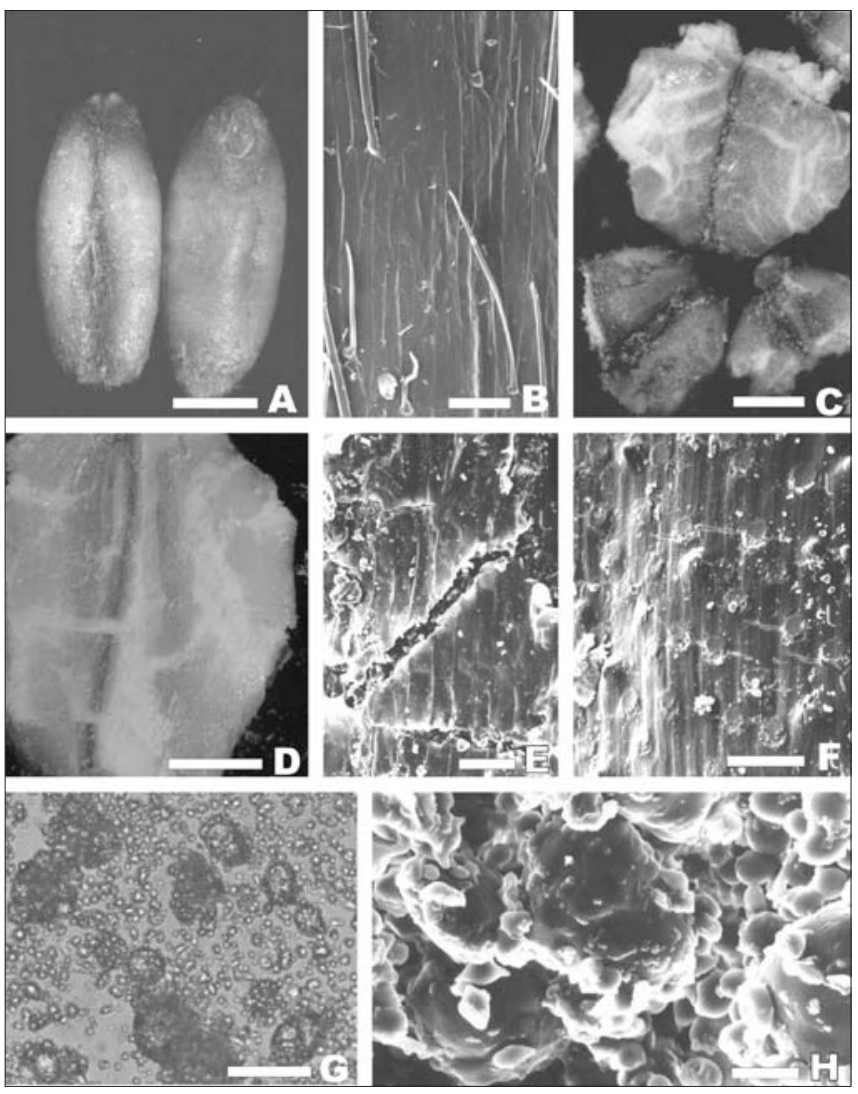

FIGURA. 2 - Avena arrollada: A, Grano desnudo y pelado para industrialización; B, superficie del grano pelado (MEB); C, avena arrollada, aspecto general; $\mathbf{D}$, avena arrollada, detalle; E, superficie del grano en la avena arrollada (MEB); F, restos de lenma en avena arrollada, muestra " $\mathrm{X}$ " (MEB); G, amiloplastos compuestos en la avena arrollada (MO); $\mathbf{H}$, detalle de los amiloplastos rodeados de gránulos de aleurona en la avena arrollada (MEB).

Barras: A, C, D: 2 mm; E, F, G: $50 \mu \mathrm{m}$; H: $10 \mu \mathrm{m}$

previo de la superficie de la cubierta seminal de los granos exigidos por la legislación vigente, configurando una falsificación.

c. el análisis micrométrico resulta adecuado para diferenciar la avena de otros cereales y, en el caso de arrojar valores de las dimensiones comprendidos en zonas no superpuestas, también para caracterización de la variedad.

Todo ello conforma parámetros que, en conjunto, permiten caracterizar los cultivares de avena estudiados, desarrollar sistemas más seguros de investigación de genuinidad en alimentos derivados, y aportar elementos para proteger la Denominación de Origen de los mismos.

\section{REFERÊNCIAS BIBLIOGRÁFICAS}

[1] NACIONES UNIDAS-CENTRO DE INFORMACIÓN, Acuerdo de la Ronda Uruguay, Acuerdo de Marrakech por el que se establece la Organización Mundial del Comercio, www.cinu.org.mx/prensa/especiales/2003/ cancun_omc/marrakech.htm, 2003.

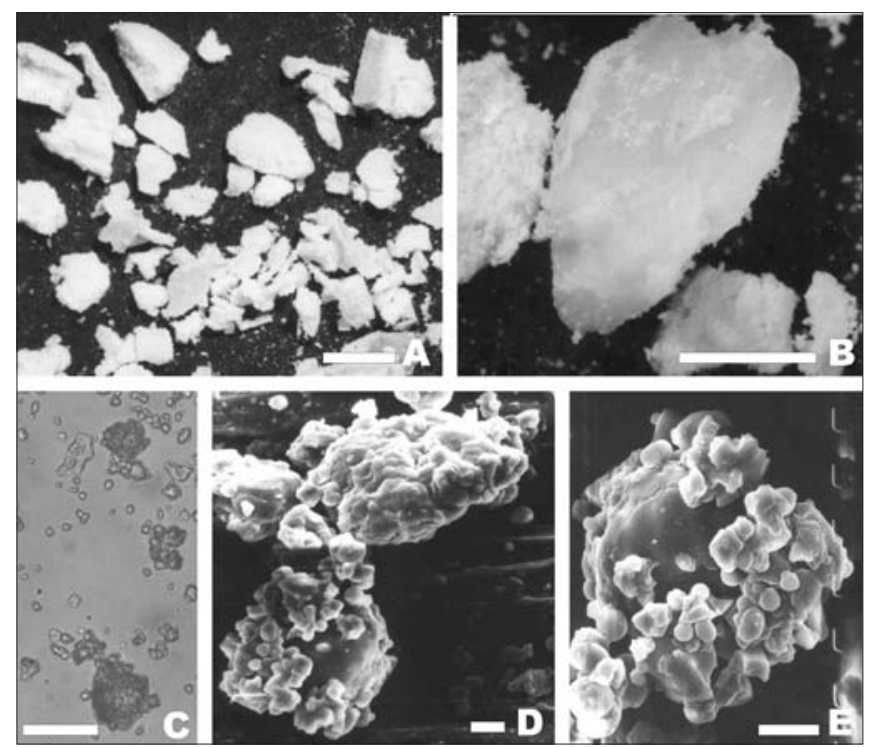

FIGURA. 3 - Salvado de avena: A, aspecto general del salvado de avena; B, detalle del salvado; $\mathbf{C}$, amiloplastos compuestos del salvado de avena (MO); D-E, detalle de los amiloplastos con gránulos presentes en el salvado de avena.

Barras: A: $2 \mathrm{~mm}$; B: $1 \mathrm{~mm}$; C: $50 \mu \mathrm{m}$; D, E: $10 \mu \mathrm{m}$

[2] DAVY, B.M.; DAVY, K.P.; HO R.C.; BESKE, S.D.; DAVRATH, L.R.; MELBY, C.L. High-fiber oat cereal compared with wheat cereal consumption favorably alters LDL-cholesterol subclass and particle numbers in middle-aged and older men. American Journal of Clinical Nutrition, v.76, p.351-358, 2002.

[3] ERAZO CASTREJON, S.; DOEHLERT, D.C.; APPOLONIA. B.L. Application of oat oil in breadbaking. Cereal Chem., v.78, p.243-248, 2001.

[4] PATON, D.; BRESCIANI, B. A.; HAN, N. F.; HART, J. (1995) Oats: Chemistry, technology and potential uses in the cosmetic industry. Cosmetics and Toiletries, v.110, p.63-70, 1995.

[5] BYE, C.; FOWLE, A.S.E.; LETLEY, E.; WILKENSON, S. Lack of effect of Avena sativa on cigarette smoking. Nature, v.252, p.580-581, 1974.

[6] FDA. DEPT. OF HEALTH AND HUMAN SERVICES . Food Labeling. Table of Contents Subpart E: Specific Requirements for Health Claims. In: Code of Federal Regulations, 1997 (Revised April 1, 2003) Title 21, Vol. 2, Sec. 101.81, p. 141-145.

[7] TOMASO, C. Variedades de avena. Con los resultados a la vista. Desafío, v.21, p.14-16, 1998.

[8] SA, R.M.; DE FRANCISCO, A.; DE SOARES, F.C.T. Chemical composition of IAC 7 oats (Avena sativa L.) and influence of thermal processing on its physicochemical characteristics. Brazilian Journal of Food Technology, v.1, p.53-58, 1998.

[9] LADIZINSKY, G. A new species of Avena from Sicily, possibly the tetraploid progenitor of hexaploid oats. Genet. Resour. Plant Evol., v.45, p.1-7, 1998.

[10] WELCH, R.W.; LEGGETT, J. Nitrogen content, oil content and oil composition of oat cultivars (A. sativa) and wild Avena species in relation to nitrogen fertility, 
yield and partitioning of assimilates. Journal of Cereal Science, v.26, p.105-120, 1997.

[11] PEDO, I.; SGARBIERI, V.C. Chemical characterization of oat (Avena sativa L.) cultivars. Ciencia e Tecnologia de Alimentos, v.17, p.78-83, 1997.

[12] WAGNER, H.; BLADT, S.; ZGAINSKY, E.M. 1995. Saponin Drugs - Avenae Herba. Plant Drug Analysis, 2nd. ed. Springer-Verlag, Berlin.

[13] WANG L.Z.; WHITE P.J. Functional properties of oat starches and relationships among functional and structural characteristics. Cereal Chem., v.71, p.451458, 1994.

[14] WANG L.Z.; WHITE P.J. Structure and physicochemical properties of starches from oats with different lipid contents. Cereal Chem., v.71, p.443-450, 1994.

[15] WANG, LZ.; WHITE, P.J. Structure and properties of amylose, amylopectin, and intermediate materials of oat starches. Cereal Chem., v.71, p.263-268, 1994.

[16] FULCHER, R.G.; MILLER, S.S. Structure of oat bran and distribution of dietary fiber components. In AMERICAN ASSOCIATION OF CEREAL CHEMISTS, INC. Oat Bran, 1993. Cap.1, p.1-24

[17] AUTIO, K., MAELKKI, Y., VIRTANEN, T. Effects of processing on the microstructure of oat (Avena sativa) bran concentrate and the physicochemical properties of isolated beta-glucans. Food Structure, v.11, p.47-54, 1992.

[18] DUVE, K., WHITE, P.J. Extraction and identification of antioxidants in oats. J. AOCS 68 (1991) 365-370.
[19] KARUNAJEEWA, H.; THAM, S.H., BROUWER;J.B., BARR, A.R. Lipid and fatty acid composition of oat (Avena sativa) cultivars grown in three Australian states. Journal of the Science of Food and Agriculture 48 (1989) 339-345.

[20] INCAP - INND. Tablas de Composición de los Alimentos para Uso en América Latina. Buenos Aires, 1961.

[21] FAO. Tablas de Composición de Alimentos para Uso Internacional, Roma 1949.

[22] CRIVARO, N.O.; GUALDIERI, P., CALlONI, S. Caracterización físico-química de frutillas de dos provincias argentinas. Información Tecnológica, v.10, p.79-88, 1999.

[23] CRIVARO, N.O., CARRON, R. Determinación de cremogenados de naranja en bebidas sin alcohol por análisis micrográfico cuantitativo. Anal. Bromatol., v.XLII p.151-165, 1990.

[24] CRIVARO, N.O.; CARRON, R., NAJERA, M. Caracterización de la genuinidad del jugo comercial de naranja por análisis regresivo y micrográfico. Anal. Bromatol., v.XXXVII, p.293-305, 1985.

[25] FLINT, O., 1996. Microscopía de Alimentos. Ed. Acribia, S.A. Zaragoza.

[26] D’AMBROGIO DE ARGÜESO, A. 1986. Manual de Técnicas en Histología Vegetal. Ed. Hemisferio Sur S.A., Buenos Aires.

[27] CÓDIGO ALIMENTARIO ARGENTINO (actualizado). Ed. De la Canal y Asoc. Buenos Aires. 